\title{
Review of: "Early life stress during the neonatal period alters social play and Linel during the juvenile stage of development"
}

\author{
Ismael Maganga-Bakita ${ }^{1}$, Troy Richter $^{1}$, Richard Hunter $^{1}$ \\ 1 University of Massachusetts Boston
}

Potential competing interests: The author(s) declared that no potential competing interests exist.

Mobilization of LTR and non-LTR transposable elements have been implicated in several human diseases (Bundo et al. 2014; Li et al., 2015; Mager and Stoye, 2015; Saeliw et al., 2018). LINE1, an autonomous nonLTR, has by far attracted the most attention. A reason for that is it seems to be the only retrotransposon established to be both present and fully active in humans. Preclinical research into retrotransposons like L1 could then tell us a great deal about its role and impact in human conditions. A long line of research has established a role for retrotransposons in genetic disorders and cancer, as well as major players in genome evolution (Fedoroff, 2012; Hunter, 2020; Kazazian \& Moran, 2017). However, the degree to which L1 and its kin play a role in neurodevelopmental and psychiatric diseases has only recently begun to be studied (Daskalaskis et al, 2018; Muotri et al., 2010; Bundo et al.,2014; Doyle et al.,2017; Lapp and Hunter, 2019) and it is now clear that regulation of this TE by environmental factors may entail both major risks to neurodevelopment as well as enhanced flexibility (Erwin et al., 2014). This paper is one of a small number which have thus far grappled with the functional consequences of developmental retrotransposon activity in the brain.

The study explored the implication of stress and LINE1, in the development of social behavior during the juvenile stage, comparing between sexes. More specifically, it looked at the effect of early life stress, using an ethological-based model of predator odor exposure, on the expression of the retrotransposon LINE1 open reading frames (ORF) 1 and 2 in the juvenile male and female brain. Using RT-qPCR, they examined ORF 1 and 2 DNA and mRNA copies. ORF1 encodes an RNA-binding protein that has a chaperone activity. ORF2 encodes both an endonuclease and a reverse transcriptase. L1 relies upon these autonomously produced RNA transcripts to translocate back into target sites within the genome. Therefore, both reading frames are necessary for successful retro-transposition activity. The level at which L1 is inserted back in the DNA is thought to be more indicative of genomic instability and potential pathology than the level of RNA transcript. It is commendable that they looked at both. In the amygdala, they observed a significant effect of stress on ORF1 DNA copy number along with a sex difference. Females showed significantly higher L1 ORF1 and ORF2 DNA copy number than males. In the juvenile hippocampus, there was a significant sex difference in L1 ORF1 with males expressing lower DNA copy number than females. In the prefrontal cortex, they found that male showed more L1 ORF1 DNA copies than females. As far as mRNA 
copy number is concerned, stress and sex had no effect in the hippocampus and prefrontal cortex. Nevertheless, there was a sex difference in L1 ORF1 in the amygdala with males expressing more mRNA copies than females. Lastly, they examined a correlation between L1 ORF1 DNA copy numbers with the frequency of social play in the amygdala and prefrontal cortex. They found a significant negative correlation in the amygdala. There was no correlation in the prefrontal cortex. The study was well conducted, and the findings add to our growing knowledge. However, some limitations remain.

One limitation here is the stress-non-responsive period which is characteristic of the neonatal rodent. We know that the offspring relies exclusively on the mother's stress response system during pregnancy, which can put the unborn at risk from maternal insults and cortisol secretion. It is not until about a week or two that the neonate's hypothalamic-pituitary-adrenal system mature, and a significant neuroendocrine response is observed (Schapiro et al, 1962; S Levine et al, 1967; Hary L et al, 1981) when exposed to a stressor. As it appears from the results that stress did not consistently have significant effects in these rather highly stress-sensitive regions it would be warranted to look at later developmental timepoints when treating the rats and compare across different postnatal stages. The authors briefly and carefully made note of this.

Another important avenue for consideration in future investigations of these stress-related epigenetic modulations is the relation to the HPA system. When we examine stress models, it would be useful to consider the extent to which our stress response system may be involved. So, we may ask how does the stress-response machinery interact with the epigenetic cellular machinery in engendering resilience or pathology? Next generation sequencing techniques have helped providing some answers to such question. Our previous work in rodents shows that acute stress and corticosterone treatment generate alterations in H3K9 methylation at B2 SINE, and other retrotransposons loci in the hippocampus (Hunter et al, 2012; Bartlett, 2020). This could suggest a regulatory activity of GR on these TEs in response to stress. Lastly, in terms of sex difference, females are known to have a stronger response to stress as well as to be more susceptible to stress-related psychiatric disorders than men (Holden, 2005), and as we have noted elsewhere, sex steroid interactions with the transposome are likely to play a role here as well (Hunter 2014, Hunter 2016, Lapp and Hunter 2019). Again, looking at a possible sex difference in the stress system-epigenetic machinery interplay with transposable elements would provide more insight into both psychiatric disorders and neurological development.

In conclusion, this study conducted by Cuarenta and colleagues not only adds to the growing evidence of the effect that environmental stressors can have on the genome integrity and structure, but also connects this effect to a behavioral endpoint. The work raises a number of interesting questions about the regulatory role of retrotransposons like L1 in the development and expression of the brain and behavior that opens the door to much future research.

References:

Bartlett, Andrew A. “Corticosteroid-Dependent Regulation of retrotransposable Elements." Order No.28258216 University of Massachustetts Boston, 2020. Ann Arbor: ProQuest. Web. 9. Nov. 2021. 
Bundo, M., Toyoshima, M., Okada, Y., Akamatsu, W., Ueda, J., Nemoto-Miyauchi, T., Sunaga, F., Toritsuka, M., Ikawa, D., Kakita, A., Kato, M., Kasai, K., Kishimoto, T., Nawa, H., Okano, H., Yoshikawa, T., Kato, T., Iwamoto, K., 2014. Increased L1 retrotransposition in the neuronal genome in schizophrenia. Neuron 81, 306-313. https://doi.org/10.1016/j.neuron.2013.10.053.

Daskalakis, Nikolaos P., et al. “Noncoding RNAS: Stress, Glucocorticoids, and Posttraumatic Stress Disorder." Biological Psychiatry, vol. 83, no. 10, 2018, pp. 849-865., https://doi.org/10.1016/j.biopsych.2018.01.009.

Doyle, G.A., Crist, R.C., Karatas, E.T., Hammond, M.J., Ewing, A.D., Ferraro, T.N., Hahn, C.-G., Berrettini, W.H., 2017. Analysis of LINE-1 elements in DNA from postmortem brains of individuals with schizophrenia. Neuropsychopharmacology 42, 2602-2611. https://doi.org/10.1038/npp.2017.115.

Erwin, J. A., Marchetto, M. C., \& Gage, F. H. (2014). Mobile DNA elements in the generation of diversity and complexity in the brain. Nature Reviews Neuroscience, 15(8), 497-506. https://doi.org/10.1038/nrn3730 Fedoroff, N. V. (2012). Presidential address. Transposable elements, epigenetics, and genome evolution. Science (New York, N.Y.), 338(6108), 758-767. https://doi.org/10.1126/science.338.6108.758 Hary, L., Dupouy, J. P., \& Chatelain, A. (1981). Pituitary response to bilateral adrenalectomy, metyrapone treatment and ether stress in the newborn rat. Neonatology, 39(1-2), 28-36.

Holden, C. (2005). Sex and the suffering brain. Science, 308(5728), 1574.

Hunter, R. G. (2020). Stress, Adaptation, and the Deep Genome: Why Transposons Matter. Integrative and Comparative Biology, icaa050. https://doi.org/10.1093/icb/icaa050

Hunter, R. G., Gagnidze, K., McEwen, B. S., \& Pfaff, D. W. (2015). Stress and the dynamic genome: Steroids, epigenetics, and the transposome. Proceedings of the National Academy of Sciences, 112(22), 6828-6833. https://doi.org/10.1073/pnas.1411260111

Kazazian, H. H., \& Moran, J. V. (2017). Mobile DNA in Health and Disease. The New England Journal of Medicine, 377(4), 361-370. https://doi.org/10.1056/NEJMra1510092

Lapp, Hannah E., and Richard G. Hunter. “Early Life Exposures, Neurodevelopmental Disorders, and Transposable Elements." Neurobiology of Stress, vol. 11, 2019, p. 100174.,

https://doi.org/10.1016/j.ynstr.2019.100174.

Lapp, H. E., \& Hunter, R. G. (2019). Early life exposures, neurodevelopmental disorders, and transposable elements. Neurobiology of stress, 11, 100174.

Levine, S., Haltmeyer, G. C., Karas, G. G., \& Denenberg, V. H. (1967). Physiological and behavioral effects of infantile stimulation. Physiology \& Behavior, 2(1), 55-59.

Li, W., Lee, M.-H., Henderson, L., Tyagi, R., Bachani, M., Steiner, J., Campanac, E., Hoffman, D.A., Geldern, G. von, Johnson, K., Maric, D., Morris, H.D., Lentz, M., Pak, K., Mammen, A., Ostrow, L., Rothstein, J., Nath, A., 2015. Human endogenous retrovirus-K contributes to motor neuron disease. Sci. Transl. Med. 7 307ra153- 307ra153. https://doi.org/10.1126/scitransImed.aac8201.

Mager, D.L., Stoye, J.P., 2015. Mammalian endogenous retroviruses. Mob. DNA III 1079-1100. https://doi.org/10.1128/microbiolspec.MDNA3-0009-2014. 
Muotri, A.R., Marchetto, M.C.N., Coufal, N.G., Oefner, R., Yeo, G., Nakashima, K., Gage, F.H., 2010. L1 retrotransposition in neurons is modulated by MeCP2. Nature 468, 443-446.

https://doi.org/10.1038/nature09544.

Saeliw, T., Tangsuwansri, C., Thongkorn, S., Chonchaiya, W., Suphapeetiporn, K., Mutirangura, A., Tencomnao, T., Hu, V.W., Sarachana, T., 2018. Integrated genome-wide Alu methylation and transcriptome profiling analyses reveal novel epigenetic regulatory networks associated with autism spectrum disorder. Mol. Autism. 9. https://doi.org/10.1186/s13229-018-0213-9.

Schapiro, S., Geller, E., \& Eiduson, S. (1962). Neonatal adrenal cortical response to stress and vasopressin. Proceedings of the Society for Experimental Biology and Medicine, 109(4), 937-941. 